\title{
Dark Matter in NGC 2974
}

\author{
A. Weijmans ${ }^{1}$, D. Krajnovićc ${ }^{2}$ T. A. Oosterloo ${ }^{3,4}$, R. Morganti ${ }^{3,4}$, \\ and P. T. de Zeeuw ${ }^{1}$ \\ ${ }^{1}$ Leiden Observatory, Leiden University, Postbus 9513, 2300 RA Leiden, the Netherlands \\ email: weijmans@strw.leidenuniv.nl \\ ${ }^{2}$ University of Oxford, Denys Wilkinson Building, Keble Road, Oxford OX1 3RH \\ ${ }^{3}$ ASTRON, Postbus 2, 7990 AA Dwingeloo, the Netherlands \\ ${ }^{4}$ Kapteyn Astronomical Institute, Postbus 800, 9700 AV Groningen, the Netherlands
}

\begin{abstract}
We present H I observations of the elliptical galaxy NGC 2974, obtained with the VLA. These observations reveal that the previously detected $\mathrm{H}$ I disc in this galaxy (Kim et al. 1988 ) is in fact a ring. Combining the flat rotation curve with the central kinematics of the ionised gas, obtained with the integral-field spectrograph SAURON, allows us to determine the mass-to-light ratio $M / L$ as a function of radius, ranging from $100 \mathrm{pc}$ in the centre to $10 \mathrm{kpc}$ at the edges of the $\mathrm{H}$ I ring. A dark halo is required to explain the observed rotation: at the outer radii $\sim 70 \%$ of the total mass is dark $\dagger$.
\end{abstract}

Keywords. galaxies: elliptical and lenticular, galaxies: neutral hydrogen, galaxies: haloes, galaxies: individual: NGC 2974

H I discs in galaxies are excellent tracers for dark matter haloes: where stellar kinematics are usually confined within 2 effective radii $\left(R_{e}\right)$, the neutral gas goes out much further. In Figure 1 we present a mass model for the elliptical galaxy NGC 2974, based upon neutral and ionised gas data obtained with the VLA and SAURON, respectively.
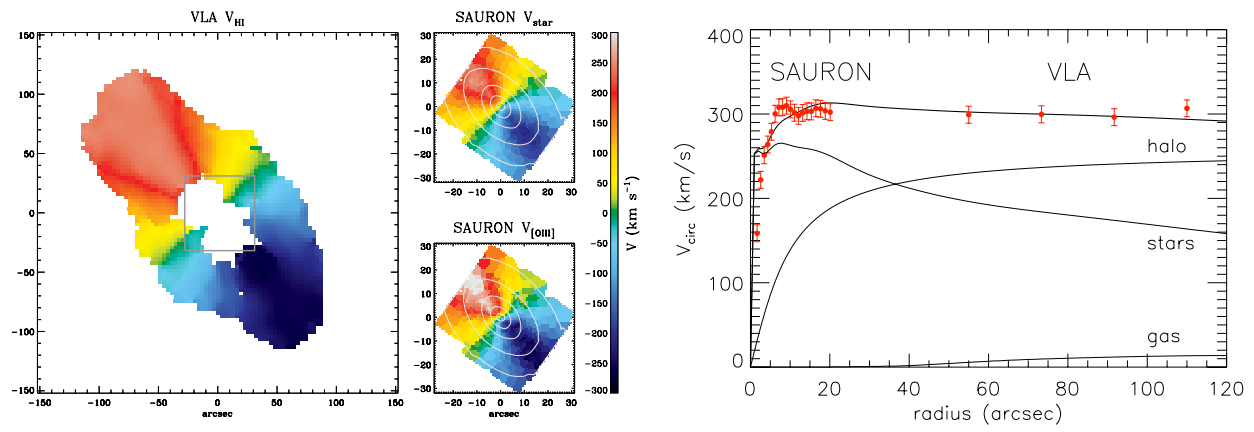

Figure 1. Left: Velocity maps of the neutral hydrogen (VLA) and stars and ionised gas (SAURON) for NGC 2974. Both the stars and the neutral and ionised gas are well aligned, suggesting that they form a single disc. Right: Circular velocity (dots) obtained with SAURON and the VLA. The best fitting model is the bold line, composed of a contribution of gas, stars and a dark halo (isothermal sphere). The VLA data extends to $5 R_{e}$.

\section{References}

Kim, D.-W., Jura, M., Guhathakurta, P., Knapp, G.R., van Gorkom, J.H., 1988, ApJ 330, 684.

$\dagger$ This poster is available at www.strw.leidenuniv.nl/ weijmans/poster_iau235_406.pdf. 